\title{
ATTENTION DEFICIT HYPERACTIVITY DISORDER SYMPTOMS IN ADULTS WITH ESSENTIAL TREMOR
}

\author{
Omer Oguzturk ${ }^{1}$, Murat Alpua ${ }^{2}$, Ersin Kasim Ulusoy ${ }^{3}$ \\ Correspondence: dr.muratalpua@yahoo.com \\ ${ }^{I}$ Department of Psychiatry, Karabuk University, Faculty of Medicine, Karabuk, Turkey \\ ${ }^{2}$ Department of Neurology, Kirikkale University, Faculty of Medicine, Kirikkale, Turkey \\ ${ }^{3}$ Department of Neurology, Kayseri Training and Research Hospital, Kayseri, Turkey
}

\section{Article History:}

Received: January 25, 2021

Accepted: September 27, 2021

Published: January 1, 2022

\section{Cite this as:}

Oguzturk O, Alpua M, Ulusoy E $K$. Attention deficit hyperactivity disorder symptoms in adults with essential tremor. Malang Neurology Journal; 2022.8:1-5. DOI:

http://dx.doi.org/10.21776/ub.mnj .2022.008.01.1

\section{ABSTRACT}

Background: Essential tremor is the most common movement disorder. İn this disease, which is characterized by tremor that increases with action and passes at rest, different accompanying symptoms can also be seen.

Objective: The purpose of this study was to investigate attention deficit hyperactivity symptoms in adults with ET.

Methods: Fifty six essential tremor patients and 56 controls were included in the study. Patients were recruited from outpatient clinic at Kirikkale University Medicine Faculty. An informed consent form was signed by each patient after detailed information. Institutional ethics committee approval was obtained. Patients' characteristics such as education level, gender, age and disease duration were recorded. Symptoms of ADHD in patients and controls were determined by using the Adult Report Deficit / Hyperactivity Disorder Self Report Scale. Short Form-36 and Hospital Anxiety and Depression Scale were also performed. Essential tremor severity was determined by using the Whiget essential tremor scale.

Results: Essential tremor patients had significantly higher rate of Adult Attention Disorder SelfReport Scale Deficit/Hyperactivity scores compared to controls. Scores of Adult Attention Deficit/Hyperactivity Disorder Self-Report Scale were associated with depression and anxiety scores and physical and mental component scores of Short Form-36 in bivariate analyses. There was a positive correlation between tremor severity and ASRS scores( ASRS A scores $=17,3 \pm 5,5, \mathrm{p}=0,032$, ASRS B scores $=27,7 \pm 6,7, p=0,043$, ASRS $\mathrm{T}$ scores $=45 \pm 12,2, \mathrm{p}=0,017)$ however there was no significant statistical relationship between the duration of disease and ASRS scores.

Conclusion: This study showed that ADHD symptoms can be observed in adult essential tremor patients and this may be associated with increased psychosocial morbidity and lowered quality of life in ET patients.

Keywords: Attention deficit and hyperactivity, essential tremor

\section{Introduction}

Essential Tremor (ET) is the most common movement disorder. Its estimated prevalence is $1 \%$ worldwide and its prevalence over 60 years old is about $5 \%$. Essential tremor usually starts as an asymmetrical postural tremor, and spreads to the opposite side over time. Tremor can only occur in a special position. When the angle of the position changes, the severity of the tremor may also differ. As a rule, it increases with action and passes at rest, but it can be seen at rest, especially when tremor severity is high. Often tremor is benign, progresses slowly, can fluctuate and do not shorten the life span. Essential tremor can be on any body part (hands, head, legs, vocal cords). Eating, drinking and writing may deteriorate when dominant in hands. In the advanced stages of the disease, voluntary movements can be quite limited due to excessive tremor. Neurological examination other than tremor is often normal. Over many years, ET was considered to be a disease characterized by only action tremor in clinical practice. Recent studies have shown that this disease may have symptoms other than action tremor and these symptoms include cognitive deficits, behavioral symptoms, cerebellar symptoms and different personality traits. ${ }^{1,2}$ Besides that, some studies showed that ET may exhibit clinically relevant psychopathology. These psychopathologies are cognitive deficits, anxiety, depression and different personality traits. ${ }^{3}$ In addition, some studies have shown that there may be attention deficit in ET as similar to Parkinson's disease. ${ }^{4}$

Attention deficit hyperactivity disorder (ADHD), a neurodevelopmental disease characterized by inadequate and inappropriate attention, impulsivity and motor hyperactivity, which is started in childhood, is still persistent in adulthood. ${ }^{5}$ It is the most frequently diagnosed mental illness in children. The prevalence rate of ADHD is approximately $2.5 \%-4.9 \%$ in the general adult population. ${ }^{6}$ The frequency of boys is higher than girls, and the ratio of boys/girls is reported between 3-5/1. The etiology of ADHD is not fully known. As with other psychiatric diseases, bio-psycho-socio-cultural causes are thought to 
play a role in the emergence of ADHD. ADHD is a clinical diagnosis. Clinician's diagnostic tools are interviews with family and child, clinical observation, physical and neurological examination, behavior assessment scales and cognitive tests. This disorder disrupts the quality of life and can occur with many other diseases especially other psychiatric disorders and adult ADHD is an important risk factor for lifelong psychiatric comorbidity, high rates of comorbidity in adults with ADHD, and studies have shown that it causes higher treatment costs. Besides that adult ADHD can accompany neurological diseases such as multiple sclerosis and migraine and it has been shown that it impairs the quality of life when it is associated with these diseases. But to our knowledge there has been no study to investigate the relationship between ET and adult ADHD.

Although the tests and scales used are not pathognomonic, they help in diagnosis. Various screening tests are used to screen for adult attention deficit and hyperactivity disorder and one of them is the Adult Attention Deficit/Hyperactivity Disorder Self-Report Scale (ASRS) and it is referring to the DSM-IV criteria for ADHD. ${ }^{7}$ It is a screening tool widely used in international epidemiological studies translated into many languages, also in Turkish. There is also a validity and reliability study of the Turkish form of ASRS. ASRS contains questions that evaluate 18 symptoms based on DSM-IV. The scale has two subscales, each consisting of nine questions, namely "attention deficit" and "hyperactivity/impulsivity". The questions are directed at determining how often each symptom appeared in the past six months. It should be kept in mind that the scale is only a screening tool and it cannot be used for diagnosis alone. The objective of our current study was to investigate attention deficit hyperactivity symptoms in adults with ET using ASRS.

\section{Methods}

Patients Patients with ET who presented to the neurology outpatient clinic were included in the study. Patients were recruited from outpatient clinic at Kirikkale University Medicine Faculty. All ET patients were diagnosed based on the diagnostic criteria from the International Parkinson and Movement Disorder Society. The principles of the current version of the declaration of Helsinki were taken into account; institutional ethics committee approval was obtained and the course of the investigation was explained to the ET indivuals and healthy controls in detail. An informed consent form was signed by each patient after detailed information.

We included 56 adults with clinically diagnosed ET and 56 healthy controls in this study. An informed consent form was signed by each patient after detailed information. Institutional ethics committee approval was obtained. Considering our outpatient clinic population, the sample size of our study was selected. Education levels, age, and sex matched volunteers without a history of psychiatric and /or neurodegenerative diseases were included in control group.

We recorded characteristics of patients such as disease duration, age, gender and education level.
We excluded patients with the following diseases and conditions; history of neurodegenerative diseases, major depression, bipolar disorder, anxiety disorder, psychosis, epilepsy, mental retardation, and substance abuse.

The Self-Report Scale of Adult Attention Deficit/Hyperactivity Disorder (ASRS) includes subscales of impulsivity, hyperactivity and inattention. Each subscale consist of 9 items and there are 18 questions in total. The first 6 questions constitute part A of the scale while the other 12 questions constitute part B. The ASRS is a rating scale which rated between 4 and 0: $4=$ very often, $3=$ often, $2=$ rarely, $1=$ sometimes, $0=$ never. These items used by the clinicians are used to determine how often a symptom has occurred over the period of past 6 months. ${ }^{7}$ The Turkish version of reliability and validity of the ASRS was performed by Dogan et al. ${ }^{8}$ The scale considered highly likely to have ADHD which individuals with a total score of 36 or greater on the ADHD scale.

In this study, we used Short Form Health Survey (SF-36), Version 1.0 to examine health-related quality of life. Role limitations arising from physical health problems, health areas of physical functioning, vitality, general and mental health, body pain, social functioning, role limitations arising from emotional problems can be measured by SF36. These areas can be grouped into two summary scores in the form of physical and mental health summary scores. ${ }^{9}$

We used Hospital Anxiety and Depression (HAD) scale to detect depression and anxiety levels of the patients. There are 14 items related to anxiety (7 items) and depression (7 items) in the scale. A total score is between 0 and 21. Scores of each item are between 3 and 0 . The cut-off point is $8 / 21$ for depression and anxiety. ${ }^{10}$

Various scales are used for essential tremor severity and one of them is Whiget essential tremor scale. ${ }^{11}$ In this study, we used this scale to rate essential tremor. The Whiget scale is a rating scale which rated between 3 and $0: 3=$ severe tremor, $2=$ moderate tremor, $1=$ mild tremor, $0=$ no tremor.

\section{Statsitical Analysis}

Version 17.0 of SPSS was uesd to analyze the results. In all tests, A p $<0.05$ was considered statistically significant. We presented continuous variables in titer of mean and \pm SD. The normality of the distribution was assessed by using Kolmogorov-Smirnov test for all variables. We used Student's t-test for normally distributed variables and used Mann-Whitney U test for abnormally distributed variables. Chi-square test and Fisher's exact test was used for categorical parameters. We used Pearson's correlation to perform bivariate analyses.

\section{Results}

We demonstrated the characteristics of healthy controls and patients in table 1. The education levels, gender and age distribution in both groups were similar. Table 1 demonstrates that compared with controls, patients with ET had significantly higher rate of ASRS, anxiety and depressive scores. Table 1 shows that ET patients had significantly decreased mean SF-36 health summary scores. 
Table 1. The characteristics of patients and controls

\begin{tabular}{lccc}
\hline & $\begin{array}{c}\text { Patients } \\
\text { (n:56) }\end{array}$ & $\begin{array}{c}\text { Controls } \\
\text { (n:56) }\end{array}$ & P \\
\hline Age, years & $33.23 \pm 9.94$ & $30.57 \pm 7.42$ & 0.11 \\
\hline Gender F/M & $26 / 30$ & $27 / 29$ & 1.00 \\
\hline Disease duration (year) & $5.78 \pm 6.08$ & & \\
\hline Education levels & 7 & 6 & 0.38 \\
Pri.school & 32 & 26 & 0.01 \\
High school & 17 & 24 & 0.03 \\
\hline ASRSA & $11.12 \pm 4.48$ & $17.08 \pm 7.10$ & 0.02 \\
\hline ASRSB & $20.01 \pm 7.49$ & $26.14 \pm 10.28$ & $<0.001$ \\
\hline ASRST & $30.94 \pm 11.42$ & $82.31 \pm 18.24$ & $<0.001$ \\
\hline SF-36 FC & $63.98 \pm 22.99$ & $70.30 \pm 14.05$ & $<0.001$ \\
\hline SF-36 MC & $58.79 \pm 18.42$ & $8.14 \pm 3.75$ & 0.04 \\
\hline HADA & $11.89 \pm 4.65$ & $6.57 \pm 3.39$ & \\
\hline HADD & $7.91 \pm 3.69$ & & \\
\hline ASRS: Adult Attentive & & & \\
\hline
\end{tabular}

ASRS: Adult Attention Deficit/Hyperactivity Disorder Self-Report Scale, SF-36 FC: Short Form Physical Component ,SF36 MC: Short Form Mental Component, HADA: Anxiety score, HADD: Depression score, F:female, M;male, ASRSA: AdultAttention Deficit/Hyperactivity Disorder Self-Report Scale part A, ASRSB: Adult Attention Deficit/Hyperactivity Disorder Self-Report Scale part B, ASRST: Adult Attention Deficit/Hyperactivity Disorder Self-Report Scale part total score

Table 2 shows that scores of the SF-36 physical and health summary component were negatively correlated with scores of ASRS in patients with ET. Table 2 demonstrates that the anxiety and depression scores in ET patients were positively correlated with scores of ASRS.

Table 2. Correlates of ASRS scores in patients

\begin{tabular}{cccccccccccc}
\hline & \multicolumn{2}{c}{ Disease duration } & \multicolumn{2}{c}{ SF-36 - FC } & SF-36-MC & \multicolumn{2}{c}{ Anxiety score } & $\begin{array}{c}\text { Depression } \\
\text { score }\end{array}$ \\
\hline \multirow{2}{*}{ ASRST } & $\mathrm{r}$ & $\mathrm{p}$ & $\mathrm{r}$ & $\mathrm{p}$ & $\mathrm{r}$ & $\mathrm{p}$ & $\mathrm{r}$ & $\mathrm{p}$ & $\mathrm{r}$ & $\mathrm{P}$ \\
\cline { 2 - 11 } & 0.07 & 0.58 & -0.43 & $<0.001$ & -0.50 & $<0.001$ & 0.44 & $<0.001$ & 0.26 & 0.005 \\
\hline
\end{tabular}

There was a positive correlation between ASRS scores and tremor severity (Table 3).

Table 3. The relationship between tremor severity and ASRS, HAD, and disease duration

\begin{tabular}{|c|c|c|c|c|c|c|c|}
\hline & \multicolumn{2}{|c|}{ Whiget $1(n=26)$} & \multicolumn{2}{|c|}{ Whiget $2(n=27)$} & \multicolumn{2}{|c|}{ Whiget $3(n=3)$} & \multirow[b]{2}{*}{$\mathbf{p}$} \\
\hline & Mean \pm SD & $\begin{array}{c}\text { Median } \\
{[\text { Min-Max] }}\end{array}$ & Mean \pm SD & $\begin{array}{c}\text { Median [Min- } \\
\text { Max] }\end{array}$ & Mean \pm SD & $\begin{array}{c}\text { Median } \\
{[\text { Min-Max] }}\end{array}$ & \\
\hline ASRSA & $9,9 \pm 4,3$ & $8[5-20]$ & $11,6 \pm 4,1$ & $12[3-21]$ & $17,3 \pm 5,5$ & $20[11-21]$ & 0,032 \\
\hline ASRSB & $17,2 \pm 7,5$ & $14[9-35]$ & $21 \pm 6,9$ & $21[10-35]$ & $27,7 \pm 6,7$ & $31[20-32]$ & 0,043 \\
\hline ASRST & $27,2 \pm 10,7$ & $24[15-53]$ & $33 \pm 10,6$ & $33[14-56]$ & $45 \pm 12,2$ & $51[31-53]$ & 0,017 \\
\hline HADA & $11,5 \pm 4,5$ & $11,5[3-20]$ & $11,6 \pm 4,7$ & $12[4-21]$ & $17,3 \pm 2,3$ & $16[16-20]$ & 0,087 \\
\hline HADD & $7,6 \pm 3,5$ & $7,5[3-16]$ & $7,7 \pm 3,7$ & $8[0-15]$ & $12 \pm 3,6$ & $13[8-15]$ & 0,189 \\
\hline Duration & $3,6 \pm 3,3$ & $2[0,5-10]$ & $7,8 \pm 7,6$ & $5[1-30]$ & $6,7 \pm 2,9$ & $5[5-10]$ & 0,022 \\
\hline
\end{tabular}

There was no significant statistical relationship between the duration of disease and ASRS scores (Table 4).

Table 4. The relationship between disease duration and ASRS

\begin{tabular}{ccc}
\hline & Correlation coefficient & p \\
\hline ASRSA & 0,151 & 0,268 \\
\hline ASRSB & 0,152 & 0,264 \\
\hline ASRST & 0,172 & 0,206 \\
\hline
\end{tabular}




\section{Discussion}

According to our knowledge, this is the first study to investigate ADHD symptoms in adult ET patients. Previous studies have shown that although there is no cognitive impairment in ET patients, there may be attention deficit ${ }^{2}$ and although when there is no cognitive impairment, there is no absolute mechanism to explain the lack of attention in ET but our study showed that the mean ASRS scores of ET patients were significantly higher than the control group. So that this may indicate that attention deficit may originate from comorbidity of ADHD in ET patients.

In general, essential tremor (ET) is considered to be a benign disease however many studies showed that the quality of life in ET is significantly affected by several factors.

Tremor severity, comorbid depression, and cognitive impairment are the most important factors affecting the quality of life in ET. ${ }^{12}$ Previous studies showed that quality of life is impaired in patients with ADHD. ${ }^{13}$ In our study, there is a negative correlation between SF-36 scores and ADHD scores. These results indicate that patients with ET have a deterioration in the quality of life due to the increase in ADHD scores.

The results of our study showed that ASRS scores increased when tremor severity increased. An increase in ASRS scores with tremor severity without any other cognitive impairment may indicate that tremor alone would impair attention and this finding may indicate that attention deficit in essential tremor is not only comorbidity of ADHD. Beside that, our study showed that there was no increase in ASRS scores due to increased disease duration and this finding may indicate that tremor severity affects attention regardless of disease duration in ET.

Attention deficit / hyperactivity disorder is significantly related with several psychiatric comorbidities at any age of onset. ${ }^{14}$ Depression and anxiety disorders, personality disorders and substance abuse are the most common comorbid psychopathologies in ADHD. In our study, patients with ET had higher anxiety, and depression scores, and these findings were consistent with the previous studies.

Depression scores and symptoms were at high rates in ET patients. Besides, depression was identified as a risk factor in the development of ET. Researchers suggested that depression was not a result of the disease but apart of the fundamental mechanism of the disease. ${ }^{15}$

In addition, anxiety scores and symptoms were at high rates in ET patients as well. It has been shown that both physical and mental quality of life deteriorates in patients with ET. In addition to the effects related to tremor in daily life, anxiety and depression were also found to be effective in the deterioration of the quality of life. ${ }^{16}$

According to our knowledge, the only study showing the relationship between neurodegenerative diseases and ADHD is the Multiple sclerosis (MS) study previously conducted by our study group. This study showed that MS patients have more ADHD symptoms than healthy controls. $^{17}$

It is not known why ADHD scores in ET patients are high. The importance of the cerebellum in the pathogenesis of ET was showed in previous studies. Duan et al showed that more severe inattention symptoms were associated with lower gray matter volume in cerebellar tonsil and culmen. ${ }^{18}$ Influence of cerebellum may be the common mechanism of both diseases. Another mechanism may be accompanied by psychiatric comorbidities in both diseases besides that comorbid ADHD may be the only responsible for the lack of attention in ET patients. However, the pathogenesis of attention deficit in ET is still unclear. From this point of view, further comprehensive researches are needed to explain the pathogenesis.

There was a limitation in our study. This limitation is as a result of the small number of patients.

Since ET is a very common disease, these findings should be reviewed with further studies with a higher number of patients. In this respect, further comprehensive studies could give more valuable information in the future so we think that further larger studies will confirm these results.

\section{Conclusion}

In conclusion, our study suggested that symptoms of attention deficit and hyperactivity symptoms may occur in adults with ET and these symptoms may be caused by comorbid ADHD. In this respect, ASRS can be used practically in ET patients and patients with suspected ADHD may be referred to psychiatry for further evaluation.

\section{Conflict of Interst}

There is no conflict of interest.

\section{Acknowledgement}

We thanks to our families for their support.

\section{References}

1. Jankovic J. Essential tremor: a heterogenous disorder. Mov Disord; 2002. 17(4):638-44.

DOI: $10.1002 / \mathrm{mds} .10221$.

2. Medeiros LM, de Castro PC, Felício AC, et al. Patients with essential tremor can have manual dexterity and attention deficits with no impairments in other cognitive functions. Arq Neuropsiquiatr; 2016. 74(2):122-7. DOI: 10.1590/0004-282X20160006.

3. Chandran V, Pal PK. Essential tremor: beyond the motor features. Parkinsonism Relat Disord; 2012. 18(5):407-413. DOI:10.1016/j.parkreldis.2011.12.003.

4. Lafo JA, Jones JD, Okun MS, et al. Memory similarities between essential tremor and Parkinson's disease: A final common pathway? The Clinical Neuropsychologist; 2015. 29(7), 985-1001. DOI: 10.1080/13854046.2015.1118553.

5. Dopheide JA, Pliszka SR. Attention-deficithyperactivity disorder: An update. Pharmacotherapy.2009;29:656-679.

DOI: 10.1592/phco.29.6.656.

6. Simon V, Czobor P, Balint S, et al. Prevalence and correlates of adult attention-deficit hyperactivity disorder: Meta-analysis. Br J Psychiatry; 2009. 194:204-211. DOI: 10.1192/bjp.bp.107.048827. 
7. Kessler RC, Adler LA, Gruber MJ, et al. Validity of the World Health Organization Adult ADHD SelfReport Scale (ASRS) Screener in a representative sample of health plan members. Int $\mathbf{J}$ Methods Psychiatr Res; 2007. 16:52-65.

DOI: $10.1002 / \mathrm{mpr} .208$.

8. Dogan S, Oncu B, Varol-Saracoglu G, et al. Validity and reliability of the Turkish version of the Adult ADHD Self-Report Scale (ASRS-v1.1). Anatolian Journal of Psychiatry; 2009. 10:78-87. Avalaible from: https://alpha-psychiatry.com/en/validity-andreliability-of-the-turkish-version-of-the-adult-adhdself-report-scale-asrs-v1-1-131977

9. Ware JE, Sherbourne CD. The MOS 36-Item ShortForm Survey (SF-36). I. Conceptual framework and item selection. Med Care; 1992. 30:473-483. Avalaible from: https://pubmed.ncbi.nlm.nih.gov/1593914/

10. Bjelland I, Dahl AA, Haug TT, et al. The validity of the hospital anxiety and depression scale. An update literature review. J Psychosom Res; 2002. 52(2):6977. DOI: 10.1016/s0022-3999(01)00296-3.

11. Heldman DA, Jankovic J, Vaillancourt DE, et al. Essential tremor quantification during activities of daily living. Parkinsonism Relat D; 2011. 17, 537542. DOI: 10.1016/j.parkreldis.2011.04.017.

12. Chunling $\mathrm{W}$, Zheng $X$. Review on clinical update of essential tremor. Neurol Sci; 2016. 37:495-502. DOI: $10.1007 / \mathrm{s} 10072-015-2380-1$.
13. Lensing MB, Zeiner P, Sandvik L, et al. Quality of life in adults aged 50+ with ADHD. Journal of Attention Disorders; 2015. 19, 405-413.

DOI: $10.1177 / 1087054713480035$.

14. Lin YJ, Yang LK, Gau SS. Psychiatric comorbidities of adults with early- and late-onset attentiondeficit/hyperactivity disorder. Aust N Z J Psychiatry.; 2016. 50(6):548-56.

DOI: $10.1177 / 0004867415609423$.

15. Louis ED, Benito-Leo'n J, Bermejo- Pajera F, Neurological Disorders in Central Spain (NEDICES) Study Group. Selfreported depression and antidepressant medication use in essential tremor: crosssectional and prospective analyses in a populationbased study. Eur J Neurol; 2007. 14:1138-1146.

DOI: $10.1111 /$ j.1468-1331.2007.01923.x.

16. Chandran V, Pal PK. Quality of life and its determinants in essential tremor. Parkinsonism Rel Disord; 2013. 19(1):62-65.

DOI: 10.1016/j.parkreldis.2012.06.011.

17. Alpua M, Turkel Y, Gunes HN, et al. Attention deficit/hyperactivity disorder in adults with multiple sclerosis. Int J Ment Health Psychiatry; 2017. 3:3. DOI: $10.4172 / 2471-4372.1000147$.

18. Duan K, Chen J, Calhoun VD, et al. Neural correlates of cognitive function and symptoms in attentiondeficit/hyperactivity disorder in adults. Neuroimage Clin; 2018. May 2;19:374-383.

DOI: 10.1016/j.nicl.2018.04.035. 